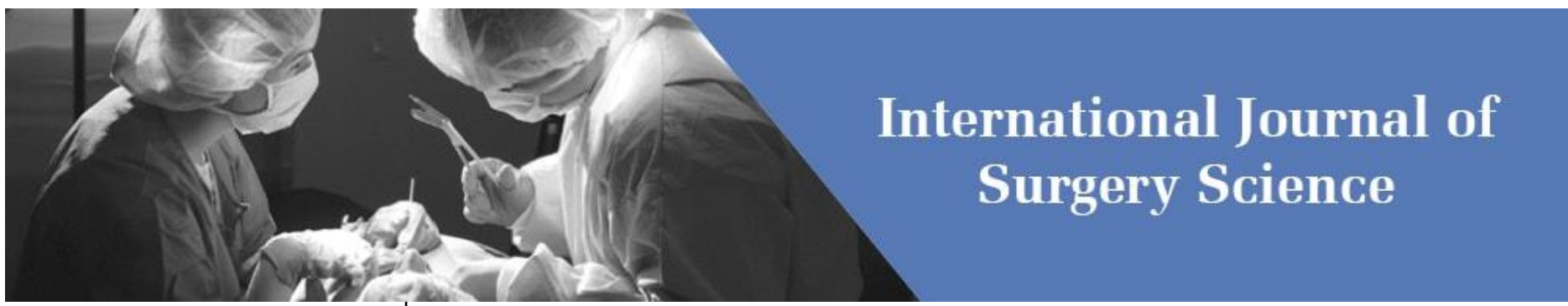

E-ISSN: 2616-3470

P-ISSN: 2616-3462

(C) Surgery Science

www.surgeryscience.com

2019; 3(3): 423-426

Received: 05-07-2019

Accepted: 25-07-2019

Dr. Janga Jayaram

Associate Professor, Department of General Surgery, Kurnool Medical

College, Kurnool, Andhra Pradesh, India

Dr. Sreeram Seshadri

Senior Resident, Department of General Surgery, Kurnool Medical College, Kurnool, Andhra Pradesh, India

Dr. Sai Praneeth Reddy

1 st Year Post Graduate,

Department of General Surgery,

Kurnool Medical College, Kurnool,

Andhra Pradesh, India

\section{Clinical study and management of acute intestinal obstruction}

\section{Dr. Janga Jayaram, Dr. Sreeram Seshadri and Dr. Sai Praneeth Reddy}

DOI: $\underline{\text { https://doi.org/10.33545/surgery.2019.v3.i3g.655 }}$

\section{Abstract}

Background: The etiology of acute intestinal obstruction, which is one of the commonest surgical emergencies, varies between countries, and has also changed over the decades ${ }^{1}$.The current study aims to review the various causes, clinical features and the outcome of surgical procedures in relation to etiological factors in 50 intestinal obstruction patients admitted at Government General Hospital, Kurnool (Oct 2014 Oct 2016)

Methods: This was a prospective study of 50 patients admitted with a diagnosis of acute intestinal obstruction at GGH, Kurnool between Oct 2014-Oct 2016. Data related to the objectives of the study were collected and analysed by comparing with various standard studies.

Results: Acute intestinal obstruction was the diagnosis in $3 \%$ of all patients admitted, with a M: F ratio of 1.38:1. The commonest age group affected was 31-40 years. In our patients, the main cause of obstruction was obstructed hernia (36\%), due to lack of awareness among the rural population. Adhesions and bands (26\%) were the second most common cause of intestinal obstruction followed by sigmoid volvulus (12\%), TB abdomen (8\%) and malignant obstruction (6\%). Surgery was the mainstay of treatment, with herniorraphy, adhesiolysis and resection - anastomosis being the most commonly performed procedures. Post-operative complications noted were - wound infection (12\%), respiratory infection (6\%) and prolonged ileus $(6 \%)$. In the present study of 50 cases, 5 patients (10\%) died due to septicemia and MODS. Conclusion: This study demonstrates that the pattern of intestinal obstruction differs from the Western world with obstructed hernias rather than adhesions being the most common cause. Majority of the patients need timely surgical relief of obstruction to avoid the development of peritonitis, sepsis and multi-organ failure.

Keywords: Etiology, acute intestinal obstruction, strangulation, obstructed hernia

\section{Introduction}

Bowel obstruction isdefined as obstruction in forward propulsion of the contents of the intestine either due to:

- Dynamic causes (adhesions, hernia, neoplasm),

- Adynamic / pseudo-obstruction (related to biochemical disturbances - paralytic ileus) ${ }^{[2]}$.

\section{Cardinal features of intestinal obstruction are ${ }^{[3]}$ :}

1. Colicky abdominal pain

2. Vomiting

3. Abdominal distension

4. Absolute constipation

Diagnosis of strangulation still remains a challenge. Multi-slice CT scan abdomen (with a sensitivity of $94-100 \%$ and specificity of $90-95 \%$ ) can achieve an accurate diagnosis of the site, severity and cause of obstruction; and also assess the presence of strangulation / closed loop obstruction ${ }^{[4]}$.

Clinical, radiological and operative findings put together can bring out the best and accurate diagnosis of intestinal obstruction. Bowel obstruction in young age, in unscarred abdomen and large bowel obstruction needs early surgery. Early diagnosis of obstruction, pre-operative preparation, Skillful operative management, proper surgical technique and intensive postoperative care carries a grateful result.
Corresponding Author:

Dr. Sreeram Seshadri

Senior Resident, Department of

General Surgery, Kurnool Medical

College, Kurnool, Andhra Pradesh,

India 
Mortality ranges from $3 \%$ for simple obstruction to as much as $30 \%$ when there is vascular compromise or perforation of the obstructed bowel ${ }^{[5,6]}$.

\section{Aims and Objectives}

- To study the various causes and clinical features of intestinal obstruction.

- To study the various surgical procedures and their outcomes in relation to etiological factors in intestinal obstruction patients.

\section{Patients and Methods}

The current study is a prospective analysis of 50 cases of intestinal obstruction (11-70yrs of age) admitted at Government General Hospital, Kurnool, over a period of 2 years between October 2014 to Oct 2016.

Soon after admission, history was taken and the diagnosis was established by clinical exam, often supported by radiological investigations like erect $\mathrm{X}$ ray \& USG abdomen in all cases. CECT abdomen was performed in select patients with stable hemodynamics.

Patients with clear-cut signs and symptoms of acute obstruction were managed by appropriate surgical procedure after resuscitation. Findings were recorded and photographs were taken. Surgery adopted and criteria for deciding the procedure and the post-operative course were noted. Histo-pathological examination of the specimen /biopsy was done whenever necessary.
Results: The study of 50 cases of intestinal obstruction during Oct 2014 to Oct 2016 at Government General Hospital, Kurnool is as follows:

- The incidence of acute intestinal obstruction in our hospital was $3 \%$ of total surgical cases. Intestinal obstruction was more common in the age group of 31-40 years (22\% cases), with a slight male preponderance $(\mathrm{M}: \mathrm{F}=1.38: 1)$

- Small bowel obstruction is more common than large bowel obstruction (39 vs 11)

- Pain abdomen, vomiting, abdominal distension and constipation are the four cardinal features of intestinal obstruction, present in most of the cases (80-100\%).

- $74 \%$ of the patients had significant past history of abdominopelvic surgeries like - appendectomy (8\%), hernioplasty $(26 \%)$, LSCS $(6 \%)$, TAH + tubectomy (12\%) etc.. to name a few. 3 cases had past history of tuberculosis.

Etiology: The most common etiological factor for intestinal obstruction in our study was obstructed hernia (36\%), followed by adhesions/bands (26\%), sigmoid volvulus (12\%) and TB abdomen (8\%).

- Malignant obstruction (6\%) is far more common in large bowel, than in small bowel. In large bowel, malignant obstruction is more common on the left side than the right side.

- Surgery was performed within $24-48$ hrs in majority of the patients. The selection criteria of the surgical procedure were based on intra-operative findings as depicted in the table below:

Table 1: Incidence of Different Etiologies

\begin{tabular}{|c|c|c|c|}
\hline Cause & No. of cases & Percentage & Surgery \\
\hline Obstructed hernias & 18 & $36 \%$ & Hernia repair (12) Resection anastomosis (6) \\
\hline Adhesions / bands & 13 & $26 \%$ & Adhesiolysis / Release of bands \\
\hline TB abdomen & 3 & $6 \%$ & Resection anastomosis \\
\hline Intussusception & 1 & $2 \%$ & (end to end ileo-ileal, / ileo-transverse,/ ileo-jejunal) \\
\hline Mesenteric ischemia & 3 & $6 \%$ & Diverticulectomy \\
\hline Meckel's diverticulum & 1 & $2 \%$ & \\
\hline
\end{tabular}

Table 2: Etiology of Large Bowel Obstruction

\begin{tabular}{|c|c|c|c|}
\hline Cause & No. of cases & Percentage & Surgery \\
\hline Neoplasm & 3 & $6 \%$ & Colostomy \\
\hline Volvulus & 6 & $12 \%$ & De-rotation (1) Resection anastomosis (5) \\
\hline TB Stricture & 1 & $2 \%$ & Resection anastomosis \\
\hline Intussusception & 1 & $2 \%$ & Milking / reduction \\
\hline
\end{tabular}

- Post-operative complications noted were - wound infection $(12 \%)$, respiratory infection $(6 \%)$, prolonged ileus $(6 \%)$, entero-cutaneous fistula (2\%), septicemia / deaths (10\%)

- MORTALITY: -In the present study of 50 cases, 5 patients (10\%) died due to septicemia and MODS- (2strangulated femoral hernia, 1 mesenteric vascular ischemia and 2 cases were due to rectal malignancies). Among the factors influencing the mortality and morbidity are age, state of hydration, nutritional status, viability of the bowel, etiology of obstruction, site of obstruction, delay in diagnosis and surgical intervention and associated medical illness ${ }^{[7]}$.

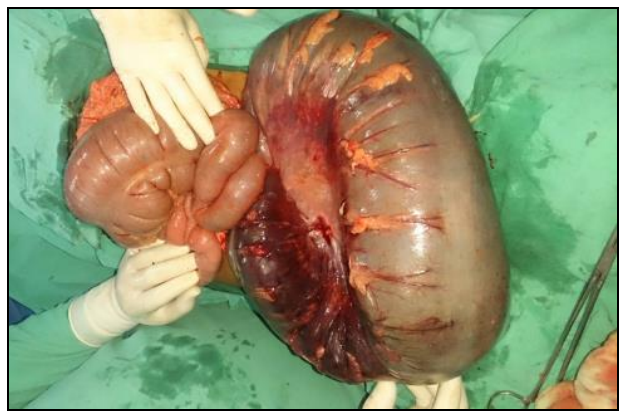

Fig 1: Gangrenous sigmoid volvulus 


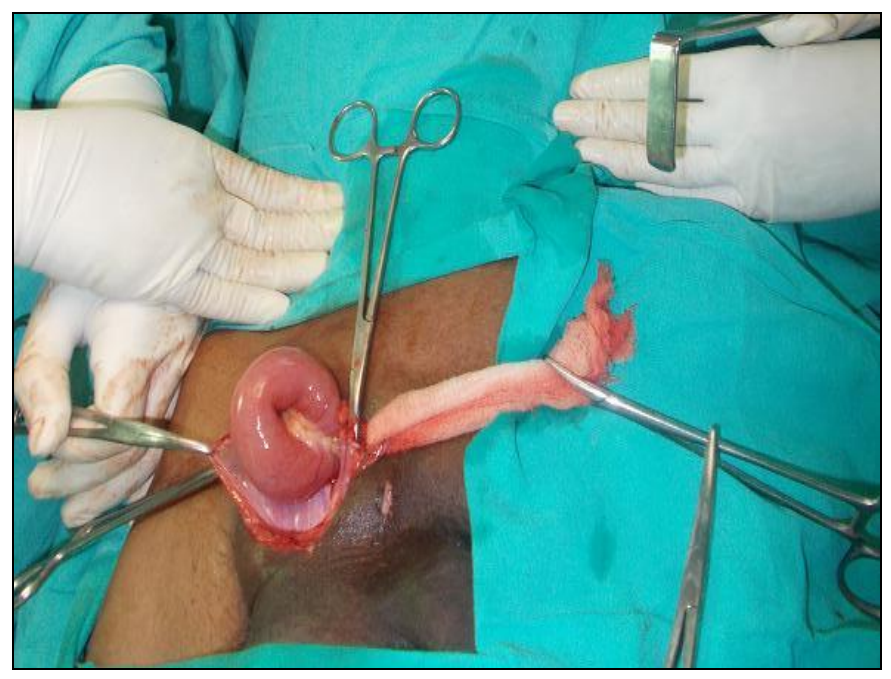

Fig 2: Obstructed inguinal hernia

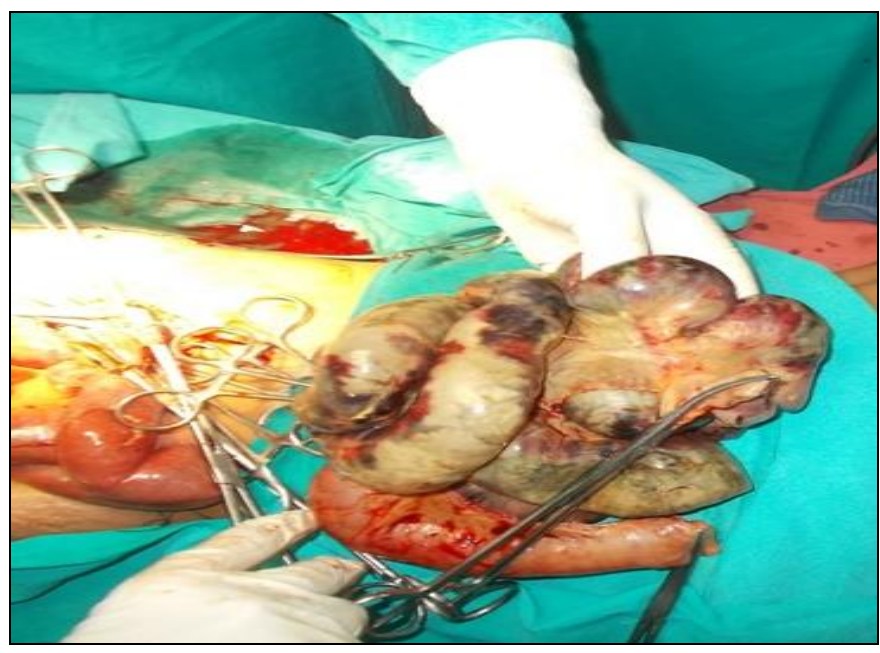

Fig 3: Mesenteric ischemia with gangrenous small bowel

\section{Discussion}

Acute intestinal obstruction continues to be a common surgical emergency (1-4\% incidence). In our study of 50 cases of intestinal obstruction at GGH, Kurnool, maximum incidence was in 31-40yrs (22\%), with aM: F ratio of 1.38:1 which is in agreement with the studies of Souvik Adhikari et al. ${ }^{[8]}$ and Jahangir Sarwar Khanseries ${ }^{[9]}$.

The etiology of intestinal obstruction varies from one country to another. In the present series, small bowel obstruction contributed to $78 \%$ and large bowel obstruction to $22 \%$ of the total cases.

The explanation for the etiological shift towards adhesions and then hernia in most Western studies is that people are seeking early treatment for hernias due to increased awareness ${ }^{[10]}$. In contrast, in developing countries like India, the commonest cause continues to be obstructed/strangulated hernia (36\% in our study, foll by adhesions $26 \%$ \& volvulus $12 \%$ ).

On review of the earlier Indian studies, only $10 \%$ of intestinal obstructions were related to adhesions. The rise in the incidence of adhesion - related obstructions (26\%) is attributed to the increased number of abdomino-pelvic surgeries ${ }^{[10]}$.

Mesenteric vascular ischemia constituted for about $6 \%$ in the present study (3 / 50 cases).The reduced incidence of mesenteric ischemia for the past few years is due to timely intervention of ischemic heart disease and its complications (Souvik Adhikari et al. $)^{[8]}$.

Other causes of intestinal obstruction in our study were -
Volvulus (12\%), TB abdomen (8\%), neoplasms (6\%) intussusception (4\%), Meckel's diverticulum (2\%) etc.

\section{Surgical management}

Adhesiolysis was done in 10 cases which included postoperative/inflammatory adhesions (20\%). Release of bands was done in 3 cases $(6 \%)$.

Anatomical hernia repair was done in 12 cases (24\%) of which 7 were inguinal hernia (Modified Bassini repair), 4 were incisional hernia and 1 was femoral hernia.

Anatomical hernia repair with resection and anastomosis was done in 6 cases $(12 \%)-2$ strangulated inguinal hernia, 2 strangulated incisional hernia and 2 strangulated femoral hernia. Resection and end-to-end ileo-ileal primary anastomosis was done in 5 cases, (10\%) which included cases of stricture (2 pts), intussusception (1pt), and mesenteric ischemia (2 pts).Resection and end-to-end jejuno-ileal primary anastomosis was done in 1 case with mesenteric ischemia.

Resection and ileo - transverse anastomosis was done in 1case of ileo-caecal TB.

Colostomy was done in 3 cases ( 2 cases of recto-sigmoid growth and 1 case of rectal growth).Resection \&anastomosis was done in 6 cases (gangrenous sigmoid volvulus -5 and TB stricture of ascending colon - 1). Intussusception milking was done in 1 case and volvulusde-rotation done in 1 case.

In our study, we had mortality rate of $10 \%$. Mortality in intestinal obstruction is high in individuals who develop strangulation / gangrene of the bowel, those who present beyond 72 hours and in those having pre-existing co-morbidities and elderly people ${ }^{[6,7]}$.

\section{Conclusion}

- Obstructed hernias were the most common etiological factor for intestinal obstruction in our study. This is attributed to the general reluctance for surgery among the rural population due to unawareness, poverty and fear.

- Clinical, radiological and operative findings put together can bring out the best and accurate diagnosis of intestinal obstruction.

- The classical clinical saying that the sun should not both set and rise on case of unrelieved intestinal obstruction is sound and should be followed

- There has been an overall decrease in mortality due to better understanding of the pathophysiology of intestinal obstruction, improvement in resuscitative and supportive treatment, in combination with aggressive surgical therapy.

\section{References}

1. Hayanga AJ, Bass-Wilkins K, Bulkley GB: Current management of small-bowel obstruction. Adv Surg 2005;39:1-33.

2. Nobie B. small bowel obstruction. http://emedicine.medscape.com/article/774140-overview.

3. Soo Y Kim, Jon B Morris. Small bowel obstruction. 6th ed. Chapter 68. In: Shackel Ford's Surgery of the alimentary tract, Charles J Yeo, ed. Philadelphia: Saunders Elsevier, 2007, pp1025-33.

4. Khaled Aly Matrawy, Mohamed El-Shazly. Intestinal obstruction: Role of multi-slice CT in emergency department. Alexandria Journal of medicine 2014;50:171178.

5. Scott G. Houghton, Antonio Ramos De la Medina, Michael G. Sarr, Maingot's Abdominal Operation, 11thed.Mc Graw Hill, 2007, 479-508. 
6. Jones RS. Intestinal obstruction. In: Sabiston DC, Jr. editor. Text book of surgery- Biological basis of modern surgical practice 20th edn. W.B. Saunders Company, 2017, 1247-54.

7. Jeremy E. Springer, MSc, Jonathan G. Bailey, M.Sc, MD. Management and outcomes of small bowel obstruction in older adult patients: a prospective cohort study

8. Souvik Adhikari, Mhd Zahid Hossein, Amitabha Das, Nilenjan Mitra, Udipta Ray. Etiology and outcome of acute intestinal obstruction: A review of 367 patients in Eastern India. The Saudi Journal of Gastroenterology 2010;16(4):285-7.

9. Jahangir Sarwar Khan, Junaid Alam, Hamid Hassan, Mohammed Iqbal. Pattern of intestinal obstruction a hospital based study. Pakistan Armed Forces Medical Journal 2007.

10. Dijkstra FR, Nieuwenhuijzen M, Reijnen MM. Recent clinical developments in pathophysiology, epidemiology, diagnosis and treatment of intra-abdominal adhesions. Scandinavian Journal of Gastroenterology. Supplement, 2000, 52-59. 